\title{
The Application of Zhongzhen Medicine in the Skin Diseases
}

\author{
Tian Ma and Bin Li* \\ Department of Dermatology, Yueyang Hospital Affiliated to Shanghai University of TCM, China
}

Submission: July 14, 2017; Published: August 07, 2017

*Corresponding author: Li Bin, Department of Dermatology, Yueyang Hospital Affiliated to Shanghai University of TCM, China, Tel: 86-21-65161782-6059; Email: 18930568129@163.com

\begin{abstract}
Zhongzhen medicine, which characteristics of the heaviness and descending is available to tranquillization with heavy preion, calmness the liver to clear out the wind and remove beverage and wart. Based on the clinical experience of the XiaShi and GuShi surgery treatment of skin diseases and the previous studies, it's confirmed that Zhongzhen medicine could cure skin diseases. Professor Li Bin [1] has sufficient experience in treatment of skin diseases by using Zhongzhen medicine. This paper is aimed at sharing enormous experience of Prof. Li Bin [1], so that to provide the reference for clinical treatment and promote further development and utilization of Zhongzhen medicine.
\end{abstract}

Keywords: Zhongzhen medicine; Skin diseases; Psoriasis

\section{Clinical Application}

\section{Trace to the source}

Professor Li Bin [1] draw the advantages from family GU and family Xia. The prescription is made up of Sichongtang. It is the important prescription of Xia Shi skin surgery, consisting of $30 \mathrm{~g}$ Magnetite, 30g Reddle, 30g Erosaria stone, 30g Concha Ostreae. These drugs are mainly ore, fossil or drug testacean. All kinds of Zhongzhen medicine in "Shennong's Classic of Materia Medica" are good medicines. Although there are apparent coldness and heat differences in these drugs for example, Fluoritum is located with warm yin energy, Oysters is located with cold yin energy and Reddle is bitterand salty. Although its nature is cold, On the whole its functional characteristics are to clam your nerves. Therefore, all kinds of Zhongzhen medicine can clam you nerves. This kind of medicine is used to treat irritability, insomnia and panic epilepsy [1,2]. As for the endogenous fire, Zhongzhen medicine can remove heat of fire. Therefore, Zhongzhen medicine can be used to treat skin diseases which are led by heart-fire hyperacivity, dysphoria, pyrophlegm and liver depression of the fire, including irritability and insomnia. The Zhongzhen medicines such as keel, oyster, red ochre, abalone shell is mainly included in the Jueyin meridian [3,4]. So, the most of Zhongzhen medicine have the effects on calming liver and restraint yang by accumulating yin and cooling the liver heat effects for example, Zhenganxifeng decoction, Lingjiaogouteng decoction and Jiangni decoction. In the process of treating skin diseases Professor
Lee found that Zhongzhen medicine can obviously alleviate the symptoms of skin itching. TCM believes that "wind" is a key pathogenesis of skin itching. The human body likes a ship sailing in the sea, the wind is coming and the boat is going to fall. Be sure to add Zhongzhen medicine on the ship. If the itching caused by wind must be pressed with a few stones, which plays a very good effect. Although it is easy to understand,there must be a reason. For example, we use calcium glucose acid to relieve itching in clinical practice. The ancients did not have calcium calcium. It is precisely that using of calcium ions in the Zhongzhen medicine, trace element by play sedative effect, that's why in the middle of clinical, antihistamine drugs have more or less a certain level of sedation [5-7]. There is a word called "mental disorder may be cured with weighty sedatives" in Chinese. Referring to use Zhongzhen drugs to treat fright timidity and insanity. If sleep is not good, then the damage to the skin is very obviously. Therefore, the use of Zhongzhen medicine may the key point in clinical treatment of skin diseases. Its more in-depth mechanism of action is also worth digging and studying.

\section{Principle of use}

As we all knows Zhongzhen medicine is cold, always eat easy to damage the spleen and stomach. But, we found in clinical under the premise of the correct way to follow the traditional Chinese medicine decoction. The development of Zhongzhen medicine embodied in adjusting emotion and improving sleep 
equality and so on. Feedback is very rarely for patients with gastrointestinal discomfort. Of course, in order to avoid the irritation of the spleen and stomach of Zhongzhen medicine, in clinical treatment according to the actual situation of the spleen and stomach can choose appropriate medicine. Professor Li Bin at the time of clinical evidence will join adlay, amomum, yam, white lentils or perilla stem to protect spleen and stomach.

According to Professor Li Bin's clinical experience at the course of application of Zhongzhen medicine there is no too many taboos. From the consideration of pulse regardless of the forceful pulse, big pulse, empty pulse can use the Zhongzhen medicine. For example, in "Treatise on Exogenous Febrile Diseases maxingshigan decoction, gypsum can relieve tangy of ephedra which is a very commonly used classic prescription. According to differences in medication habits in southern and northern of China are mainly reflected on the application of drugsabout elimiating wind to tonify blood etc. The Northern like to use ephedra and ramulus cinnamomi the Southern like using honeysuckle and isatis leaf etc. Professor Li Bin believes that the skin disease due to yang positively floating, wind-heat, moist-heat, blood-heat and stagnation of qi can lead to floating Yang. That is to say that the occurrence of skin diseases must be related to heat evil, whether it is virtual or actual heat. Even if the patient has an internal cold, the skin surface tends to have a performance of heat evil. Zhongzhen medicine is not rigidly adhering to the drug because of cold or heat, which could try to use together.

\section{Clinical experience}

Zhongzhen medicine in the clinical application is very wide. The followings are summaries of the common diseases. Hoping to be able to reach more effects Prof. Li Bin has abundant experiences in the treatment of psoriasis the research team led by summarizing the clinical experience of Shanghai skin surgery on record, according to the "Yin, Yang, Yo Nomori in the outside, Yin that also". The traditional Chinese medicine theory is the essence of acute psoriasis of blood heat syndrome is the "blood heat inside, Yang floating outside", "blood heat is the foundation of the disease, Yang floating to the sign of disease". Use of clearing heat and cooling blood, suppressing hyperactive liver and subsiding yang treatment, with specific prescriptions, with the card cut. Its basic prescription consist of concha margaritifera, magnetitum, raw oysters, red ochre, coix seed, caulis Sargentodoxae, hedyotis diffusa, rehmannia, solanum nigrum, artemisia annua, gypsumis $30 \mathrm{~g}$, paeonol, radix scutellariae, radix paeoniae alba by $12 \mathrm{~g}$, smilax, folium isatidis, all 15g, shikonin, ephedra, ramulus cinnamomi, vitex, madder, curcuma rcenyujin, all 9g, licorice root $6 \mathrm{~g}[8,9]$. Cooling blood medicine together with Zhongzhen medicine can clam down the nerves and remove heat. A large number of clinical practice and a number of related research shows that this therapy not only can significantly improve the skin lesions of psoriasis, but also can regulate the degree of depression in patients with psoriasis.
It reduces disease recurrence caused by abnormal emotion such as depression and stress, which has certain significance [10-12].

In terms of herpes zoster the mechanism of Zhongzhen medicine is actually very simple. Herpes zoster due to severe pain, sleep is not good. We mentioned before that Zhongzhen medicine can produce sleep sedative effect. What's more important is XiaShi Skin surgery thinks that this disease led by cardiosplenic toxic fire, liver depression qi junction. Such as margaritifera concha, keel, concha mauritiae, amethyst the meridian is mainly attributed to clearing heart and detoxification and liver purging fire. Modern pharmacological confirm that margaritifera concha, keel, concha mauritiae, amethyst have the effect on central nervous system. So with the paeonol, dandelion, rhizoma coptidis, Phellodendron amurense Decoction treat herpes zoster [13]. Sequela of herpes zoster neuralgia is that the waste heat is not clear; ying blood disharmony we should use the treatment of clearing heat and harmony ying.

\section{Prescription}

Caulis Lonicerae, Radix Paeoniae Rubra, Radix Paeoniae Alba, Padix Salviae Miltiorrhizae, Cortex Moutan, Angelica and Sichong soup. The modern mechanism of herpes zoster is that calcium; trace elements have the effect of regulating nerve and relieving pain. When the blood deficiency for adding nourishing blood medicine, such as angelica, radix rehmanniae, radix paeoniae alba, qi deficiency for adding astragalus, codonopsis, atractylodes to encourage vital energy promoting the evil out, blood stasis for adding peach seed, Szechwan lovage rhizoma, padix paeoniae rubra to promote blood circulation and remove blood stasis and relieve pain through pulse.

The above two kinds of diseases are the representative of Zhongzhen drug treatment. Professor Li Bin increase the amount of Zhongzhen medicine in clinical, But the actual clinical application of the drug is far more than that drugs. For the common diseases such as urticaria, eczema, etc. can play a certain degree of antihistamine effect. For some chronic, complex diseases, can also be treating by using of Zhongzhen medicine [14-19]. The ancient "chronic illness must be responsible for blood stasis chronic illness must be responsible for phlegm". In the "Treatise on Febrile Diseases" Xuanfudaizhetang, reddle toghter with inula flower treat syndrome of xiajiao phlegm. In "The Intergrating Chinese and Western Medicine", Keel oyster soup consists with Ochre, keel, oysters. Since ancient times, Zhongzhen medicine in the treatment of disease has played an important role. In the course of treatment of skin diseases, Professor Li Bin follows principles: light mental disorder, floating, movement may be cured with weighty sedatives, yin overcoming yang and yin folding yang. According to clinical needs, flexible to add and subtract, harvest the satisfaction of clinical efficacy.

\section{Acknowledgement}

This study was funded by Shanghai Science and Technology Commission to support the "Xia's surgery" genre heritage 
research base (Grant No.ZY3-CCCX-3-3033). The National Natural Science Foundation of China (Grant No.81473682). State Administration of Traditional Chinese Medicine funded the "second five" key disciplines -Chinese medicine dermatology. State Administration of Traditional Chinese Medicine funded "second five" key specialist - Chinese and Western medicine combined with skin specialist (skin disease) Shanghai Sailing Program (Grant No. 17YF1420600).

\section{Funding}

Sponsored by Chinese National Natural Science Foundation(No.81473682):Mechanisms and Functions of Impaired Nuclear Translocation of Glucocorticoid Receptor in the therapeutic effect of cleaning heat and surpressing yang;Sponsored by Shanghai Sailing Program (No.17YF1420600)

\section{References}

1. Li Su, Li Xin, Li Bin, Li Bin Blood (2014) Heat syndrome of psoriasis vulgaris treated with Jing's experience. Journal of Yunnan College of Traditional Chinese Medicine 37(5): 88-89.

2. Wu Luting, Liu Shengjin, Wu Dekang, Wang Rui, Yang Huan, et al. (2015) Research progress and clinical application of traditional Chinese mineral medicine pharmacological effects of soothing the nerves. Modern Chinese Medicine 9(17): 892-898.

3. Zhang Yi, Qiu Longshu (2012) The effectiveness of city medicine in the treatment of skin diseases. Journal of Liaoning University of Traditional Chinese Medicine 11(14): 111-112.

4. Jiang Wencheng, Li Bin (2015) Traditional Chinese medicine treatment for the treatment of refractory psoriasis. Chinese Journal of Integrative Medicine 11(35): 1288-1290.

5. Zhang Li, Li Jianwei (2012) The application of Zhongzhen medicien in the skin department. Chinese Journal of Dermatology and venereal disease 5(11): 324-325.

6. Sun DZ, Zhang X, Xu JY, Peng H, Ye Min, et al. (2015) Therapeutic effect of Jinlongshe Granule () on quality of life of stage IV gastric cancer patients using EORTC QLQ-C30: A double-blind placebo-controlled clinical trial. Chin J Integr Med 21(8): 579-586.

7. Wang Yingjie, Li Bin, Li Bin, Chai Han (2014) Experience and medication characteristics in the treatment of psoriasis. Chinese Journal of Dermatology and venereal disease 13(6): 374-376.
8. Jiang Wencheng, Ma Tian, Li Bin, Li Bin (2014) The use of tranquilizing "from the blood on the treatment of psoriasis vulgaris. 7(41): 13371340.

9. Zhang Jingyi, Li Bin, Li Bin (2013) On the treatment of allergic skin disease from blood. Shanghai traditional Chinese medicine magazine. 47(10): 17-18.

10. Wang Yuming, Wu Xiaohong, Zeng Xue, WA li Liu, Zhuang Guokang (2012) The use of drugs for treatment of skin disease of city experience. Journal of traditional Chinese Medicine 16(53): 1372-1373.

11. FAN Bin, LI Xin, ZE Kan, XU Rong, SHI Ruo-fei, et al. (2015) Expression of T-helper 17 cells and signal transducers in patients with psoriasis vulgaris of blood-heat syndrome and blood-stasis syndrome. Chin Integr Med 21(1): 10-16.

12. Tan YQ, Liu JL, Bai YP, Zhang LX (2011) Literature research of chinese medicine recipes for the treatment of psoriasis vulgaris with bloodheat syndrome type. Chin J Integr Med 17(2): 150-153.

13. Han Shirong, Wang Juan (2007) Four Decoction in the treatment of postherpetic neuralgia. Shanxi traditional Chinese Medicine 28(2): 171-172.

14. Chen XZ, Cao ZY, Liao LM, Liu ZZ, Du J (2014) Application of Serum Pharmacology in Evaluating the Antitumor Effect of Fuzheng Yiliu Decoction from Chinese Medicine. Chin J Integr Med 20(6): 450-455.

15. Xuan ML, Lu CJ, Han L, Xiang Y (2015) Circulating levels of inflammatory cytokines in patients with psoriasis vulgaris of different chinese medicine syndromes. Chin J Integr Med 21(2): 108-114.

16. Li N, Li YQ Li HY, GUO Wei, Bai YP (2012) Efficacy of externally applied chinese herbal drugs in treating psoriasis: a systematic review. Chin J Integr Med 18(3): 222-229.

17. Ekor M, Odewabi AO (2014) Occupational exposure to municipal solid wastes and development of toxic neuropathies: possible role of nutrient supplementation: Complementary and alternative medicines in chemoprevention. Chin J Integr Med 20(9): 643-653.

18. Lu XY, Xu H, Li G, Zhao T (2014) Study on correspondence between prescription and syndrome and the essence of phlegm and blood stasis syndrome in coronary heart disease based on metabonomics. Chin J Integr Med 20(1): 68-71.

19. YANG L, Li TT, CHU YT, Chen Ke, Tian SD, et al. (2016) Traditional chinese medical comprehensive therapy for cancer-related fatigue. Chin J Integr Med 22(1): 67-72.

\section{Your next submission with Juniper Publishers} will reach you the below assets

- Quality Editorial service

- Swift Peer Review

- Reprints availability

- E-prints Service

- Manuscript Podcast for convenient understanding

- Global attainment for your research

- Manuscript accessibility in different formats

( Pdf, E-pub, Full Text, Audio)

- Unceasing customer service

Track the below URL for one-step submission https://juniperpublishers.com/online-submission.php 